\title{
Relación Entre Prácticas Parentales Y El Nivel De Asertividad, Agresividad Y Rendimiento Académico En Adolescentes
}

\author{
Claudia González Fragoso, Doctora \\ Universidad Autónoma del Estado de Hidalgo, México \\ Yolanda Guevara Benítez, Doctora \\ Universidad Nacional Autónoma de México, México \\ David Jiménez Rodríguez, Doctor \\ Universidad Autónoma del Estado de Hidalgo, México
}

Raúl J. Alcázar Olán, Doctor

Universidad Iberoamericana Puebla, México

doi: 10.19044/esj.2017.v13n20p37 URL:http://dx.doi.org/10.19044/esj.2017.v13n20p37

\begin{abstract}
The behaviors that parents use to educate their adolescent children can influence various aspects of their psychosocial performance, either by promoting the development of behavioral skills or by associating with behavioral problems, aggressiveness or depressive and anxious symptoms. The objective of the study was to know the relationship of parental practices with the level of assertiveness, aggressiveness and academic performance in a sample of young Mexican adolescents. Participated 565 students of a Mexican public secondary, with an average age of 13.01 years. Two instruments, the Parental Practice Scale for Adolescents (Andrade \& Betancourt, 2008) and the Assertive Behavior Scale (Michelson \& Wood, 1982) were applied individually, in addition, the students' average school grades were obtained. Mean scores indicated aggressive behavior patterns throughout the sample, significantly higher in males ( $\mathrm{p}<.05)$. The maternal practices of Communication, Autonomy, and Behavioral Control correlated with the level of assertiveness in the young, and with their school average; both indicators, significantly higher in adolescent females compared to males. Paternal practices correlated with the level of assertiveness and aggressiveness in males.
\end{abstract}

Keywords: Parental practices, Assertiveness, Social skills, Aggressiveness, Academic performance 


\section{Resumen}

Las conductas que los progenitores emplean para educar a sus hijos adolescentes pueden influir en diversos aspectos de su desempeño psicosocial, ya sea promoviendo el desarrollo de habilidades conductuales, o bien, asociarse con problemas de comportamiento, agresividad o sintomatología depresiva y ansiosa. El objetivo del estudio fue conocer la relación que guardan las prácticas parentales con el nivel de asertividad, agresividad y rendimiento académico, en una muestra de jóvenes adolescentes mexicanos. Participaron 565 estudiantes de una secundaria pública mexicana, con edad promedio de 13.01 años. Se aplicaron individualmente dos instrumentos, la Escala de Prácticas Parentales para Adolescentes (Andrade \& Betancourt, 2008) y la Escala de Conducta Asertiva (Michelson \& Wood, 1982), además, se obtuvo el promedio de calificaciones escolares de los alumnos. Los puntajes promedio indicaron patrones de conducta agresiva en toda la muestra, significativamente mayores en los varones $(\mathrm{p}<.05)$. Las prácticas maternas de Comunicación, Autonomía y Control Conductual correlacionaron con el nivel de asertividad en las jóvenes, y con su promedio escolar; ambos indicadores, significativamente mayores en las adolescentes mujeres en comparación con los varones. Las prácticas paternas correlacionaron con el nivel de asertividad y agresividad en los varones.

Palabras-clave: Prácticas parentales, Asertividad, Habilidades sociales, Agresividad, Rendimiento Académico

\section{Introducción}

Las prácticas parentales están constituidas por las conductas que padres y madres emplean para educar a sus hijos; las más estudiadas han sido las relacionadas con el apoyo y con el tipo de control (Andrade \& Betancourt, 2008). El apoyo se refiere al grado en que los hijos se sienten aceptados e importantes para sus padres. Respecto al control, se han ubicado dos patrones o tipos: el primero es el denominado control conductual, que está relacionado con que los padres pongan límites y reglas para la conducta de sus hijos, sin que ello implique un patrón de autoritarismo o chantaje; el segundo es denominado control psicológico y se refiere a la utilización de recursos como la inducción a la culpa, la devaluación y las críticas excesivas, para imponer un estilo de conducta a los hijos.

Autores como Florenzano et al. (2011) explican que, dentro de las prácticas parentales, el control psicológico se puede observar cuando padres o madres muestran hacia sus hijos: invalidación de sentimientos, restricción de expresiones verbales, retiro de amor, inducción de culpa, control 
intrusivo, devaluación, chantaje, o agresión física y verbal; situaciones que pueden llevar a desarrollar conductas inadaptadas en sus hijos. Mientras que Andrade, Betancourt, Vallejo, Segura y Rojas (2012) mencionan que el control conductual y el apoyo parental incluyen el respeto y la comunicación entre padres e hijos; tales patrones de interacción han mostrado una relación positiva con el bienestar de los adolescentes.

Diversos autores han aportado evidencias de que las prácticas parentales difieren entre mujeres y hombres, así como en función del sexo de los hijos (Andrade et al., 2012; Benjet et al., 2009). Oliva, Parra y Aranz (2008) reportaron que los estilos parentales de las madres suelen incluir mayores muestras de afecto, y que son ellas quienes promueven más la autonomía y el control conductual en sus hijos. Triana, Ávila y Malagón (2010) explican que las prácticas de crianza y su justificación dependen de la cultura, así como del nivel socioeconómico y educativo de los padres, dado que se encuentran involucrados tres procesos psicosociales: las practicas, las pautas y las creencias. Según el estudio realizado por González, Molina, Montero y Martínez (2013), el grado de escolaridad de ambos padres influye sobre sus prácticas de crianza, así como sobre el grado de apoyo y supervisión que ejercen con sus hijos, en particular con sus hijas, lo que puede ser explicado porque los padres con mayor educación tienen mayores expectativas y aspiraciones académicas para ellas. Este hallazgo, sin embargo, no ha sido consistente en otras investigaciones que analizan la influencia del grado de escolaridad parental (Pulido, Castro-Osorio, Peña \& Ariza-Ramírez, 2013).

La importancia de estudiar las prácticas parentales reside en la posibilidad de encontrar el grado de relación que existe entre éstas y diversos aspectos del desarrollo psicosocial de los niños y adolescentes. Algunas investigaciones en el campo (Andrade et al., 2012; Neves, Xavier, Vieira \& Rubin, 2013) han reportado que las prácticas parentales adecuadas pueden influir en sentido positivo, promoviendo el desarrollo de habilidades conductuales. Y también, que algunas prácticas parentales inadecuadas se asocien con agresividad y problemas psicosociales en niños y adolescentes. A continuación se exponen algunas evidencias reportadas, en ambos sentidos.

Neves et al. (2013) llevaron a cabo una investigación con 205 padres y cuidadores y 289 niños brasileños con un promedio de 10.5 años de edad. Encontraron tres predictores de agresividad en los niños: altos niveles de rechazo parental, escaso apego materno y ser varón; así mismo, el bajo apego del padre se asoció con aislamiento social infantil. García, García y Casanova (2014) realizaron un estudio con 326 estudiantes españoles con edades entre 10 y 16 años; encontraron que algunas prácticas parentales 
negativas, tales como crítica, rechazo y formas de disciplina rígida, fueron predictores de conductas de ira y agresividad física y verbal. Dicha relación se acentuó conforme los niños avanzaban en edad, por lo que fue más clara en los adolescentes.

Por su parte, Andrade et al. (2012) exploraron la relación entre las prácticas parentales y la sintomatología depresiva, en 1934 adolescentes mexicanos. Los jóvenes que percibieron mayores niveles de apoyo y comunicación, autonomía y control conductual por parte de ambos padres, mostraron menor sintomatología depresiva. Así mismo, se identificó que las prácticas maternas influyeron más que las paternas, y en las jóvenes en mayor medida que en los varones.

En otro estudio (Florenzano et al., 2011) se identificaron los estilos parentales asociados con la ideación suicida en 2346 adolescentes con edad promedio de 15.6 años. Los participantes con menores indicadores de ideación suicida fueron aquellos que percibían que sus padres les mostraban aceptación, amabilidad, afecto físico y monitoreo, además de permitirles participar en las decisiones familiares.

La investigación de Vite y Pérez (2014) refiere que el uso del castigo por parte de los padres correlacionó con la frecuencia de problemas de comportamiento en sus hijos adolescentes. Mientras que Cabrera, González y Guevara (2012) reportaron que el estrés parental y el trato rudo desencadenan la agresión, pero el monitoreo no intrusivo hace menos probable la presencia de agresividad en los adolescentes. También Nunes, Faraco y Vieira (2013) reportaron que un alto control psicológico parental fue predictor de problemas infantiles de tipo externalizado, asociados con agresividad y delincuencia, y de tipo internalizado, que incluyen excesivo control de impulsos, ansiedad y depresión.

Álvarez (2010) aclara que un patrón conductual parental es inadecuado cuando está caracterizado por rechazo, frialdad, indiferencia, disciplina basada en el castigo, o inconsistente, así como por la falta de reforzamiento de conductas prosociales, y todo ello se asocia con el hecho de que el niño emplee la agresividad al relacionarse con otras personas, dado que la agresividad es una forma de interacción aprendida. Según Gaeta y Galvanovskis (2009), entre los predictores de síntomas psicopatológicos en adolescentes y jóvenes, pueden ubicarse bajos niveles de: integración social, conductas cooperativas, habilidades sociales y estabilidad emocional, así como la presencia de impulsividad extrema.

Al considerar todas las evidencias en el campo, queda claro que las prácticas parentales pueden relacionarse con la presencia de patrones psicopatológicos en los niños y adolescentes, muy probablemente porque tales patrones implican un bajo nivel de auto-regulación y de habilidades 
sociales, asociados con actitudes negativas e incluso con conductas de riesgo (Herrera \& Repetto, 2014).

Las habilidades sociales son el conjunto de conductas que permiten a la persona desarrollarse en un contexto individual o interpersonal (Caballo, Salazar \& Equipo de Investigación CISO-A España, 2017). Un conjunto de conductas y estrategias que son aprendidas y que involucran tres componentes: conductual, cognoscitivo y emocional o afectivo (Vega, González, Anguiano, Nava \& Soria, 2009). El principal componente de las habilidades sociales es la asertividad, definida como la capacidad de expresar sentimientos positivos, de experimentar empatía y de hacer cumplidos, aspectos que reditúan en la integración social (García, Cabanillas, Morán \& Olaz, 2014). En contraparte, los problemas en las relaciones interpersonales se caracterizan por una evitación del contacto social con otros o por mantener relaciones violentas a través de un estilo de interacción inhibido o agresivo (Betina \& Contini, 2011).

Autores como Carbonero, Martín, Monsalvo y Valdivieso (2015) han reportado fuertes correlaciones entre el nivel de competencia social de los alumnos y su rendimiento académico. Pero pocos son los estudios dirigidos a explorar la relación entre rendimiento académico, nivel de habilidad social y prácticas parentales.

El estudio de Nunes et al. (2013) indicó que los niños pueden mostrar mayor agresividad que las niñas, y que, independientemente del sexo, los problemas de conducta se asocian con bajo rendimiento escolar. Betina y Contini (2011) reportaron que un bajo desempeño escolar suele asociarse con problemas de comportamiento y falta de habilidades sociales. Mientras que Malander (2016) aporta evidencia de que las prácticas parentales pueden asociarse con las estrategias y los hábitos de estudio que desarrollan los adolescentes, incluyendo actitudes, motivación, administración del tiempo, manejo de ansiedad, concentración, selección de ideas principales y preparación de exámenes, todo lo cual impacta en su rendimiento académico.

El objetivo del presente estudio fue conocer la relación que guardan las prácticas parentales con el nivel de asertividad, agresividad y rendimiento académico, en una muestra de jóvenes adolescentes mexicanos.

\section{Método}

\section{Tipo de estudio}

Descriptivo y correlacional.

\section{Participantes}

La muestra estuvo conformada por 565 estudiantes de secundaria (54.3\% de sexo femenino), con un promedio de edad de 13.01 años ( $D E=$ 
.74), inscritos en seis grupos de primer grado (49.7\%) y seis grupos de segundo, en una escuela pública localizada en un municipio del Estado de Hidalgo, México.

\section{Instrumentos de evaluación}

Para la medición de variables se utilizaron dos escalas.

I. Escala de Prácticas Parentales para adolescentes: PP-A (Andrade \& Betancourt, 2008). La escala se diseñó para ser aplicada a adolescentes mexicanos, entre 12 y 18 años de edad, para valorar las prácticas que perciben en sus progenitores. Se obtuvo su validez y confiabilidad con estudiantes de escuelas públicas de la Ciudad de México. Consta de 80 reactivos, 40 relacionados con las prácticas del padre y 40 relativos a las prácticas de la madre, agrupados en nueve dimensiones. Las cuatro dimensiones paternas son: Comunicación y control conductual, con 16 reactivos (alfa=.97), mide la comunicación entre el padre y el adolescente así como el conocimiento que tiene el padre de las actividades de sus hijos (ejemplo de reactivo: habla conmigo sobre mis problemas); Autonomía con ocho reactivos (alfa=.94), mide el respeto que muestra el padre de las decisiones que toman los hijos (p.e. Respeta mi punto de vista, aunque sea diferente del que él dice); Imposición con ocho reactivos (alfa=.90), mide el grado en que el padre impone sus creencias y conductas al hijo (p.e. Él cree que tiene la razón en todo), y Control psicológico con ocho reactivos (alfa=.90), mide inducción de culpa, devaluación y críticas excesivas al adolescente (p.e. Me culpa por cualquier cosa). Las dimensiones correspondientes a la madre son: Comunicación con 11 reactivos (alfa=92), mide la comunicación entre la madre y el adolescente (p.e. Habla conmigo sobre mis problemas); Autonomía con siete reactivos (alfa=.86), mide el respeto que muestra la madre a las decisiones que toman los hijos (p.e. Apoya mis decisiones); Imposición con ocho reactivos (alfa=.81), mide el grado de imposición de creencias y conductas (p.e. Quiere que sea como ella es); Control psicológico con ocho reactivos (alfa=.80), mide inducción de culpa, devaluación y críticas excesivas (p.e. Me hace sentir que soy un/a inútil), y Control conductual con siete reactivos (alfa=.84), mide el conocimiento que tiene la madre sobre las actividades del hijo (p.e. Conoce lo que hago por las tardes después de la escuela).

Cada reactivo tiene cuatro opciones de respuesta, se asigna 1 a la opción de respuesta nunca, 2 a la opción algunas veces, 3 a muchas veces y 4 a siempre. Los puntajes obtenidos en cada dimensión se dividen entre el número de reactivos de ésta; los mayores puntajes indican que el adolescente percibe con más frecuencia la presencia de esa dimensión.

II. Escala de Conducta Asertiva para Niños, CABS (Michelson \& Wood, 1982). Se utilizó la adaptación de Lara y Silva (2002), quienes 
estandarizaron la escala para población infantil y adolescente mexicana, en un rango de edad de 9 a 17 años, obteniendo una consistencia interna de .80 . Está compuesta por 27 reactivos con cinco opciones de respuesta, divididos en dos factores. El primer factor se denomina Dar y recibir cumplidos y críticas, tiene 20 reactivos que evalúan si el niño o adolescente se comporta de manera asertiva, pasiva o agresiva, en su interacción con diversas personas y situaciones sociales [ejemplo de reactivo: Un niño o un adulto te dice “creo que eres una persona agradable”. Lo que normalmente dirías es: a) No, no soy tan agradable, b) ¡Sí, creo que soy el mejor!, c) Gracias, d) No le dices nada pero te da pena, e) Gracias, realmente soy fabuloso]. El factor 2 se denomina Enojo y culpa, cuenta con siete reactivos que evalúan las reacciones ante situaciones que le son molestas [p.e. Un niño o un adulto te hace algo que no te gusta y esto te hace enojar mucho. Lo que normalmente harías es: a) Le gritas ¡eres odioso!, b) Le dices estoy enojado contigo, no lo vuelvas a hacer, c) Te sientes dolido por lo que hizo pero no le dices nada a la persona, d) Le dices estoy enojado ¡no me caes bien!, e) Ignoras lo que te hizo y no le dices nada a la persona].

La escala se califica asignando a cada respuesta un valor de 0 a 5 puntos, para caracterizar el comportamiento como asertivo (con un puntaje total entre 27 y 42), pasivo (entre 43 y 50) o agresivo (entre 51 y 135). El estilo asertivo se refiere a respetar los derechos propios y los de los demás; el pasivo se caracteriza por conductas de indefensión, sometimiento y culpa, que impiden defender los derechos propios e implican la aceptación de lo que otros pidan; el estilo agresivo corresponde a conductas y actitudes que signifiquen agredir a los demás sin tomarlos en cuenta. Las calificaciones de la escala se interpretan de manera inversa: a menor puntaje, mayor el grado de asertividad y habilidades sociales; a mayor puntaje mayor grado de agresividad.

III. Cuestionario de datos sociodemográficos. Se recabaron por escrito datos sobre diversos aspectos de la vida familiar del niño. En el presente reporte se retoman sólo los datos obtenidos referentes al grado de escolaridad de ambos progenitores (Ejemplo de reactivo: ¿Cuál es el último grado de estudios de tu mamá? Con las siguientes opciones de respuesta: primaria, secundaria, bachillerato, carrera técnica, licenciatura y posgrado).

La medida de rendimiento académico de los alumnos fue obtenida al final del ciclo escolar, considerando el promedio de calificaciones en las materias de Español, Matemáticas y Ciencias Naturales.

\section{Procedimiento}

La investigación fue autorizada por las instituciones escolares correspondientes. El director de la escuela secundaria así como el Consejo 
Técnico aprobaron la aplicación de los instrumentos, un mes antes de concluir el ciclo escolar. Se aclaró a los alumnos que su participación era voluntaria y que podían dejar de responder en el momento que lo desearan, sin consecuencia alguna. Ningún estudiante se rehusó a contestar los cuestionarios.

La aplicación de los instrumentos fue llevada a cabo por estudiantes y egresados de la carrera de psicología, quienes previamente recibieron capacitación por parte de los investigadores. Cuatro evaluadores se presentaron en cada grupo para solicitar la colaboración del alumnado, darles instrucciones y repartir los cuestionarios. Los alumnos respondieron individualmente los instrumentos; conforme concluían levantaban la mano permaneciendo sentados y uno de los evaluadores recogía los formatos. Cada profesor estuvo presente durante los 50 minutos asignados para la sesión de aplicación a su grupo. Al concluir la sesión se agradeció la participación de los alumnos.

\section{Análisis de datos}

Todos los datos fueron capturados en el programa IBM-Statistical Package for the Social Sciences (IBM-SPSS, versión 20 para Windows). Los instrumentos fueron calificados por los investigadores. Se obtuvieron los puntajes individuales para cada instrumento y los promedios escolares de los alumnos. Tales datos fueron agrupados por sexo de los participantes, para realizar la comparación de las medias a través de la Prueba t de Student, a excepción de la variable nivel académico de los progenitores, donde la comparación se realizó a través de la prueba U de Mann Whitney. Se obtuvo el coeficiente de correlación de Pearson para conocer la relación entre las variables evaluadas: 1) prácticas parentales de padres y madres, 2) asertividad de los adolescentes, y 3) rendimiento académico (promedio escolar). Finalmente, se obtuvo el coeficiente de correlación de Spearman para conocer la relación entre el nivel académico de los progenitores y el resto de las variables.

\section{Resultados}

La Tabla 1 muestra las medias obtenidas en las variables de estudio. Los resultados se presentan separando los datos de los participantes por sexo, para dar cuenta de las diferencias en función de dicha variable.

La percepción de las jóvenes acerca de las prácticas paternas indicó un nivel que puede considerarse medio (algunas veces) en la dimensión Comunicación y control conductual; un nivel alto (muchas veces) en la dimensión Autonomía; las dimensiones Imposición y Control psicológico se ubicaron en niveles bajos (entre nunca y algunas veces). En los varones, el patrón percibido en las prácticas paternas fue similar, aunque en la 
dimensión Comunicación y control conductual, ellos perciben niveles significativamente superiores a ellas $(M=2.69$ vs. $M=2.50 ; t=-2.44 ; p=$ $<.05)$.

En cuanto a la percepción de las jóvenes acerca de las prácticas maternas, tres dimensiones pueden considerarse en nivel alto (muchas veces): Comunicación, Autonomía y Control conductual; mientras que la dimensión Imposición se ubicó en un nivel medio (algunas veces) y Control psicológico en un nivel bajo. El patrón percibido por parte de los varones es similar, pero el nivel de Autonomía que perciben de parte de sus madres es significativamente mayor que el percibido por las mujeres $(M=2.93$ vs. $M=$ 2.79; $t=-2.37 ; p<.05)$.

Por el contrario, las jóvenes percibieron niveles significativamente mayores que sus compañeros varones en las dimensiones relativas a imposición, control psicológico y control conductual por parte de sus madres.

En la escala de asertividad, tanto varones como mujeres obtuvieron puntajes promedio que indicaron patrones de conducta agresiva $(M=54.94$; $D E=12.66 ; M=52.69, D E=12.93$, respectivamente), significativamente mayores en los varones $(t=-2.08, p<.05)$. La comparación por sub-escalas arrojó diferencias significativas en el factor 1 Dar y recibir cumplidos y críticas $(t=-2.43, p<.05$ ), lo cual fue indicativo de que los varones mostraban tendencia a comportarse de manera más agresiva que las jóvenes, al recibir una crítica, al expresar sus sentimientos, al iniciar una conversación $\mathrm{o}$ al hacer una petición. No se encontraron diferencias por sexo en lo relativo al factor 2 Enojo y culpa, porque unos y otros reaccionaban de forma predominantemente agresiva ante situaciones que les resultaban molestas.

En la variable rendimiento académico (promedio escolar), las jóvenes obtuvieron un mayor nivel que los varones $(M=8.54, D E=.85 ; M=8.03, D E$ $=.86$, respectivamente), $\mathrm{y}$ tales diferencias fueron estadísticamente significativas $(t=7.10, p<.01)$.

El nivel académico de las madres fue mayor que el de los padres, aunque la diferencia no fue estadísticamente significativa. Además, el nivel académico de las madres de los jóvenes varones fue mayor al de las madres de las adolescentes ( $U$ de Mann $=32573.00, p=.00$ ). De la misma forma, el nivel académico de los padres fue mayor en el caso de hijos varones y menor en los padres de las jóvenes ( $U$ de Mann $=31272.00, p=.02$ ). 
Tabla 1. Comparación de medias, por sexo de los participantes, en las variables evaluadas.

\begin{tabular}{cccccc}
\hline Variables & \multicolumn{2}{c}{ Mujeres } & \multicolumn{2}{c}{ Hombres } \\
\cline { 2 - 5 } Prácticas parentales & $M$ & $D E$ & $M$ & $D E$ & $t$ \\
\hline $\begin{array}{c}\text { Comunicación y Control } \\
\text { Conductual Paterno }\end{array}$ & 2.50 & .95 & 2.69 & .85 & $-2.44^{*}$ \\
\hline Autonomía Paterna & & & & & \\
\hline Imposición Paterna & 1.70 & .61 & 1.77 & .59 & -1.25 \\
\hline Control Psicológico Paterno & 1.42 & .57 & 1.34 & .46 & 1.76 \\
\hline Comunicación Materna & 2.95 & .86 & 3.02 & .72 & -1.02 \\
\hline Autonomía Materna & 2.79 & .73 & 2.93 & .63 & $-2.37^{*}$ \\
\hline Imposición Materna & 2.08 & .66 & 1.97 & .64 & $2.04^{*}$ \\
\hline Control Psicológico Materno & 1.67 & .68 & 1.54 & .53 & $2.47^{*}$ \\
\hline Control Conductual Materno & 3.18 & .69 & 3.06 & .62 & $2.16^{*}$ \\
\hline Asertividad (Habilidades sociales) & 52.69 & 12.93 & 54.94 & 12.66 & $-2.08^{*}$ \\
\hline Dar y recibir cumplidos y críticas & 34.91 & 8.53 & 36.77 & 8.84 & $-2.43^{*}$ \\
\hline Enojo y culpa & 17.77 & 5.38 & 18.16 & 5.43 & -.85 \\
\hline Promedio escolar & 8.54 & .85 & 8.03 & .86 & $7.10^{* *}$ \\
\hline
\end{tabular}

Nota: $* \mathrm{p}<.05 ; * * \mathrm{p}<.001$.

La Tabla 2 presenta el resultado de las correlaciones obtenidas entre las variables evaluadas. Las correlaciones encontradas fueron moderadas, porque se ubicaron en un nivel entre .30 y .50 (Cohen, 1988).

Ninguna práctica paterna correlacionó con el grado de Asertividad o agresividad de los jóvenes, al analizar los datos de la muestra total, ni al considerar los datos de las jóvenes participantes. Pero el análisis de los reportes obtenidos de los varones indican correlaciones negativas entre dos dimensiones de la escala de prácticas parentales y la escala de asertividad: los puntajes más altos en Comunicación/control conductual paterno y en Autonomía se asociaron con menores niveles de agresividad en los jóvenes (lo que implica más habilidades sociales asertivas). Es importante recordar que la escala de asertividad es inversa, por lo que las puntuaciones altas indican agresividad. Entonces, estas correlaciones implican que a mayor comunicación entre el padre y el adolescente, a mayor conocimiento que él tenga sobre las actividades de su hijo, y a mayor respeto que muestre por las decisiones que tome el joven, menor es el nivel de agresividad del hijo y mayor su nivel de respuestas asertivas. 
Correlaciones similares a las descritas se ubicaron entre las prácticas maternas y la asertividad, considerando los datos de la muestra total de jóvenes. Las dimensiones Comunicación, Autonomía y Control conductual correlacionaron negativamente con el resultado de la prueba de Asertividad, es decir, a mayores puntajes en esas dimensiones menores calificaciones en la escala de asertividad, lo cual indica altos niveles de agresividad. Así mismo, se encontró una relación positiva entre la dimensión Control psicológico materno y la escala de Asertividad, lo que implica que, a mayor inducción de culpa, devaluación y críticas excesivas ejercidas por parte de la madre, mayor grado de agresividad (menor asertividad) en el adolescente.

Al separar los datos de acuerdo al sexo de los participantes, las correlaciones entre las prácticas maternas y la asertividad/agresividad se siguen observando, en la misma magnitud y dirección en que fueron descritas, pero sólo para el caso de las adolescentes. Los datos de los varones indican correlación solamente entre la dimensión Comunicación materna y el total de la prueba de Asertividad.

El Control conductual materno correlacionó positivamente con el promedio escolar, pero únicamente para el caso de las jóvenes.

Tabla 2. Correlación de las prácticas parentales con: asertividad (habilidades sociales) y promedio escolar.

\begin{tabular}{|c|c|c|c|c|c|}
\hline Prácticas parentales & $\begin{array}{c}\text { Sexo } \\
\text { del joven }\end{array}$ & $\begin{array}{c}\text { Total de } \\
\text { Escala } \\
\text { Asertividad }\end{array}$ & $\begin{array}{l}\text { Dar y recibir } \\
\text { cumplidos }\end{array}$ & $\begin{array}{l}\text { Enojo y } \\
\text { culpa }\end{array}$ & $\begin{array}{l}\text { Promedio } \\
\text { escolar }\end{array}$ \\
\hline \multirow{3}{*}{$\begin{array}{c}\text { Comunicación/ control } \\
\text { conductual } \\
\text { Paterno }\end{array}$} & M & $-.303^{* *}$ & $-.242^{* *}$ & $-.313^{* *}$ & .054 \\
\hline & $\mathrm{F}$ & $-.284 * *$ & $-.269 * *$ & $-.256^{* *}$ & $.136^{*}$ \\
\hline & Ambos & $-.282 *$ & $-.244 * *$ & $-.277 * *$ & .066 \\
\hline \multirow[t]{3}{*}{ Autonomía paterna } & M & $-.309 * *$ & $-.291 * *$ & $-.247 * *$ & $.145^{*}$ \\
\hline & $\mathrm{F}$ & $-.263 * *$ & $-.249 * *$ & $-.239 * *$ & $.163^{* *}$ \\
\hline & Ambos & $-.277 * *$ & $-.258 * *$ & $-.241 * *$ & $.129 * *$ \\
\hline \multirow[t]{3}{*}{ Imposición paterna } & M & .083 & .065 & .088 & -.044 \\
\hline & $\mathrm{F}$ & $.170 * *$ & $.167^{* *}$ & $.143^{*}$ & -.060 \\
\hline & Ambos & $.135 * *$ & $.126^{* *}$ & $.120 * *$ & -.065 \\
\hline \multirow{3}{*}{$\begin{array}{c}\text { Control psicológico } \\
\text { paterno }\end{array}$} & M & $.186^{* *}$ & $.127^{*}$ & $.227 * *$ & .010 \\
\hline & $\mathrm{F}$ & $.240 * *$ & $.208 * *$ & $.246 * *$ & $-.139 *$ \\
\hline & Ambos & $.211 * *$ & $.165^{* *}$ & $.235^{* *}$ & -.053 \\
\hline \multirow{3}{*}{$\begin{array}{c}\text { Comunicación } \\
\text { materna }\end{array}$} & M & $-.369 * *$ & $-.304 * *$ & $-.364 * *$ & .040 \\
\hline & F & $-.370 * *$ & $-.335 * *$ & $-.362 * *$ & $.143^{*}$ \\
\hline & Ambos & $-.364 * *$ & $-.314 * *$ & $-.360 * *$ & .083 \\
\hline \multirow[t]{3}{*}{ Autonomía materna } & M & $-.260 * *$ & $-.219 * *$ & $-.250 * *$ & .115 \\
\hline & $\mathrm{F}$ & $-.365 * *$ & $-.343 * *$ & $-.334 * *$ & $.202 * *$ \\
\hline & Ambos & $-.311^{* *}$ & $-.277 * *$ & $-.293 * *$ & $.131 * *$ \\
\hline
\end{tabular}




\begin{tabular}{cccccc}
\hline Imposición materna & $\mathrm{M}$ & .070 & .055 & .074 & -.051 \\
& $\mathrm{~F}$ & $.285^{* *}$ & $.279^{* *}$ & $.242^{* *}$ & -.082 \\
& $\mathrm{Ambos}$ & $.177^{* *}$ & $.161^{* *}$ & $.161^{* *}$ & -.040 \\
\hline \multirow{2}{*}{$\begin{array}{c}\text { Control psicológico } \\
\text { materno }\end{array}$} & $\mathrm{M}$ & $.281^{* *}$ & $.249^{* *}$ & $.248^{* *}$ & -.073 \\
& $\mathrm{~F}$ & $.375^{* *}$ & $.357^{* *}$ & $.335^{* *}$ & $-.146^{*}$ \\
& $\mathrm{Ambos}$ & $.325^{* *}$ & $.297^{* *}$ & $.293^{* *}$ & -.082 \\
\hline $\begin{array}{c}\text { Control conductual } \\
\text { materno }\end{array}$ & $\mathrm{M}$ & $-.286^{* *}$ & $-.265^{* *}$ & $-.238^{* *}$ & $.154^{*}$ \\
& $\mathrm{~F}$ & $-.374^{* *}$ & $-.326^{* *}$ & $-.382^{* *}$ & $.305^{* *}$ \\
& Ambos & $-.341^{* *}$ & $-.305^{* *}$ & $-.321^{* *}$ & $.255^{* *}$ \\
\hline
\end{tabular}

Notas: $\mathrm{M}=$ masculino; $\mathrm{F}=$ femenino; ${ }^{*} p<.05 ;{ }^{* *} p<.001$.

Por ultimo, el análisis de Spearman indicó que el nivel académico de los progenitores no se correlacionó con sus prácticas parentales, y tampoco con el nivel de asertividad y el rendimiento académico de sus hijos adolescentes.

\section{Conclusión}

Los resultados de esta investigación, aunados a los hallazgos de estudios previos, hacen claro que las prácticas parentales son un factor muy importante para el desarrollo psicosocial de los niños y adolescentes.

Los trabajos de diversos autores han aportado evidencias de que las prácticas parentales caracterizadas por bajos niveles de comunicación y excesivo control psicológico se relacionan con problemas psicopatológicos, sintomatología depresiva e ideación suicida (Florenzano et al., 2011; Gaeta \& Galvanovskis, 2009), así como con conducta agresiva en niños y adolescentes (García et al., 2014; Neves et al., 2013; Vite \& Pérez, 2014). Además, se sabe que el nivel de agresividad suele ser mayor en chicos que en chicas (Nunes et al., 2013) y que las prácticas parentales que las madres ejercen suelen tener mayor impacto que las de los padres (Andrade et al., 2012). Los datos generados en el presente estudio confirman lo anterior, pero también aportan datos nuevos.

El patrón de prácticas parentales fue distinto en padres que en madres, y también fue distinto en función del sexo de su hijo.

Tanto los datos de las mujeres como los de los varones indicaron que sus padres sólo mostraron un nivel alto en la dimensión Autonomía, con niveles bajos de Imposición y Control psicológico, y un nivel medio de Comunicación y control conductual (dimensión relacionada con que los padres pongan límites y reglas para la conducta de sus hijos). Este patrón puede ser indicativo de que los padres siguen asumiendo que la educación de los hijos, en general, recae principalmente en las madres, creencia fuertemente arraigada en amplios círculos de la sociedad mexicana. Incluso puede explicar el hecho de que no se hayan encontrado correlaciones entre 
las prácticas paternas y las medidas de asertividad y rendimiento académico de los hijos, considerando la muestra total de participantes.

La comparación por sexo de los jóvenes permitió ver que los padres establecen más comunicación y control conductual con sus hijos varones que con sus hijas, lo que puede guardar relación con afinidades de género. El análisis estadístico confirmó que las dimensiones paternas relativas a comunicación y autonomía sólo correlacionaron con el nivel de habilidades sociales (asertividad/agresividad) de los varones, aunque no con su rendimiento académico.

Al considerar todos los datos relacionados con las prácticas paternas puede decirse que, en general, entablan poca comunicación con sus hijos adolescentes, y que a menor grado de comunicación y conocimientos que ellos tengan sobre las actividades de los chicos menor es el nivel de habilidad social de éstos, por lo que muestran mayor agresividad.

El patrón de prácticas maternas fue distinto. Considerando la muestra total de jóvenes, dicho patrón estuvo caracterizado por un nivel alto de Comunicación, Control conductual y Autonomía, un nivel medio de Imposición y un nivel bajo de Control psicológico. Pero al analizar diferencialmente dichas prácticas, se encuentra que las madres, al parecer, conceden mayor autonomía a los varones que a las chicas y respetan más sus decisiones. Sobre las hijas, en cambio, parecen ejercer un mayor grado de imposición, control conductual (poner límites y reglas) y control psicológico (inducción de culpa, devaluación y críticas); el nivel de comunicación no mostró diferencias por sexo de los jóvenes participantes.

El análisis mostró correlaciones en el sentido esperado: los aspectos positivos como la comunicación, el control conductual y el respecto a la autonomía de los adolescentes, por parte de sus madres, correlacionó con mayor nivel de habilidades sociales (asertividad), mientras que el control psicológico, que se considera negativo, se asoció con mayor agresividad. Relaciones que se observaron con los datos de la muestra total, y que confirman que las prácticas positivas pueden considerarse factores protectores para los adolescentes, porque les permiten desarrollar habilidades para expresar sentimientos positivos, experimentar empatía, hacer y recibir cumplidos, así como otras habilidades sociales que favorecen su integración en distintos contextos, incluido el escolar (García et al., 2014). En contraparte, la ausencia de prácticas parentales positivas, así como la presencia de imposición y control psicológico sobre los adolescentes, puede propiciar patrones de comportamiento agresivo (Cabrera et al., 2012; Nunes et al., 2013).

El análisis diferencial indicó que los niveles de asertividad/agresividad de las adolescentes continuaban correlacionándose 
con las cuatro dimensiones de las prácticas maternas, pero en los varones sólo se ubicó esta correlación para la dimensión comunicación.

El conjunto de datos relacionados con las prácticas maternas son indicativos de que, si bien los niveles de comunicación parecen ser iguales, se trate de un hijo o de una hija, las madres parecen asumir un mayor control hacia el comportamiento de las chicas, tanto en un sentido positivo (control conductual) como negativo (imposición y control psicológico). Este patrón diferencial materno puede explicar, al menos parcialmente, por qué los varones, al tener menos límites y reglas, muestran mayores niveles de agresividad que las mujeres, y también puede explicar por qué el rendimiento académico es menor en ellos, dado que el control conductual materno fue la única dimensión parental que se correlacionó con el rendimiento académico.

En el caso de las jóvenes, los niveles de agresividad pueden ser explicados por la dimensión Control psicológico que fue mayor hacia ellas que hacia los varones. La inducción de culpa, devaluación y críticas ejercidas por parte de la madre pueden relacionarse con los bajos niveles de asertividad en las adolescentes (Andrade et al., 2012; Florenzano et al., 2011).

La explicación de por qué las madres ejercen este tipo de prácticas con sus hijas, en mayor medida que con sus hijos varones -quienes, además viven con mayor autonomía- debe aludir nuevamente a los estereotipos asociados al rol de género, en los cuales se espera que las mujeres se ajusten a determinadas pautas conductuales y culturales, en la mayoría de las ciudades de provincia en México. El contexto social y cultural en que se desenvuelven los adolescentes y sus padres juega un papel importante, porque influye sobre su vida familiar y sus prácticas de crianza, que se van transmitiendo de generación en generación, a partir de creencias y actitudes (Infante \& Martínez, 2016).

Por todo lo anterior, es importante que se generen estrategias de intervención, encaminadas tanto al desarrollo de habilidades sociales en los adolescentes como a mejorar las prácticas parentales de madres y padres. Tales programas de intervención pueden ser realizados en distintos contextos, aunque el ámbito escolar puede ser el idóneo para ello. Es necesario que las familias asuman pleno conocimiento de que la adolescencia es una etapa decisiva para el aprendizaje y la práctica de habilidades sociales, y que su grado de desarrollo tendrá un impacto en el ajuste psicológico, académico y social durante la vida adulta (Betina \& Contini, 2011).

Los programas de intervención deben estar dirigidos a promover el diálogo y los acuerdos entre sus integrantes, condición necesaria para que los hijos desarrollan conductas positivas y de auto-regulación, que repercuten en diversos espacios, como el escolar (Álvarez, 2010); así mismo, han de 
encaminarse al desarrollo de habilidades sociales y asertividad, que mejoran el desempeño académico y disminuyen los problemas de comportamiento (Dos Santos \& Viana, 2016).

Además de habilidades de diálogo, los progenitores requieren desarrollar mejores pautas de control conductual y apoyo positivo hacia sus hijos, tener conocimiento de las actividades que realizan, sean varones o mujeres. Estas prácticas fungen como factores de protección, porque los jóvenes que tienen poca supervisión parental tienen mayor riesgo de ser influenciados particularmente por otros jóvenes que ejercen un liderazgo negativo y promueven conductas desviadas y peligrosas.

Para concluir, es importante señalar algunas limitaciones de la presente investigación. Por un lado, los datos relativos a las prácticas parentales se obtuvieron a partir de una evaluación unilateral, la percepción de los adolescentes, pero no se tuvo un punto de comparación emanado de reportes de los propios padres. Por otra parte, los niveles de habilidad social de los jóvenes (asertividad/agresividad) fueron obtenidos a través de una escala de auto-reporte, pero no se evaluaron a través de otras fuentes.

La sugerencia para estudios futuros es que se incluyan medidas adicionales de habilidades sociales, ya sea por observación, nominación de pares, cuestionarios aplicados a profesores, padres o pares, para contar con medidas sobre la percepción que tienen otros acerca de las habilidades sociales, asertividad y agresividad de los adolescentes. Con ello se puede tener un panorama más completo de los aspectos aquí reportados.

\section{References:}

1. Álvarez, M. (2010). Prácticas educativas parentales: autoridad familiar, incidencia en el comportamiento agresivo infantil. Revista Virtual Universidad Católica del Norte, 31, 253-273. Disponible en: http://revistavirtual.ucn.edu.co/index.php/RevistaUCN/article/view/4 4

2. Andrade, P., \& Betancourt, D. (2008). Prácticas parentales: una mediación integral. En S. Rivera, R. Díaz-Loving, I. Reyes, R. Sánchez \& M. Cruz (Eds.). La psicología social en México (Vol. XII, pp. 561-565). México: Asociación Mexicana de Psicología Social.

3. Andrade, P., Betancourt, D., Vallejo, A., Segura, B., \& Rojas, R. M. (2012). Prácticas parentales y sintomatología depresiva en adolescentes. Salud Mental, 35(1), 29-36. Disponible en: http://www.scielo.org.mx/scielo.php?script=sci_arttext\&pid=S018533252012000100005

4. Benjet, C., Borges, G., Medina-Mora, M. E., Méndez, E., Fleiz, C., Rojas, E., \& Cruz, C. (2009). Diferencias de sexo en la prevalencia y 
severidad de trastornos psiquiátricos en adolescentes de la Ciudad de México. Salud Mental, 31, 155-163. Disponible en: http://new.medigraphic.com/cgi-

bin/resumen.cgi?IDREVISTA=81\&IDARTICULO=20589\&IDPUB LICACION $=2087$

5. Betina, A., \& Contini, N. (2011). Las habilidades sociales en niños y adolescentes. Su importancia en la prevención de trastornos psicopatológicos. Fundamentos en Humanidades, XII (23), 159-182. Disponible en: http://www.redalyc.org/articulo.oa?id=18424417009

6. Caballo, V., Salazar, I., \& Equipo de Investigación CISO-A España (2017). Desarrollo y validación de un instrumento para la evaluación de las habilidades sociales: "el cuestionario de habilidades sociales" (CHASO). Behavioral Psychology, 25(1), 5-24. Disponible en: http://www.academia.edu/32792649/DESARROLLO_Y_VALIDAC I\%C3\%93N_DE_UN_NUEVO_INSTRUMENTO_PARA_LA_EVA LUACI\%C3\%93N_DE_LAS_HABILIDADES_SOCIALES_EL_CU ESTIONARIO_DE_HABILIDADES_SOCIALES_CHASO_1

7. Cabrera, V., González, M., \& Guevara, I. (2012). Estrés parental, trato rudo y monitoreo como factores asociados a la conducta agresiva. Universitas Psychologica, 11(1), 241-254. Disponible en: http://revistas.javeriana.edu.co/index.php/revPsycho/article/viewFile/ 842/1533.

8. Carbonero, M., Martín, L., Monsalvo, E., \& Valdivieso, J. (2015). School performance and personal attitudes and social responsability in preadolescents students. Anales de Psicología, 31(3), 990-999. doi:10.6018/analesps.31.3.181161.

9. Cohen, J. (1988). Statistical power analysis for the behavioral sciences (2nd Ed.). Hillsdale, NJ: Erlbaum.

10. Dos Santos, L. \& Viana, M. (2016). Habilidades Sociais, Comportamentos e Desempenho Acadêmico em Escolares antes e após Intervenção. Psico-USF, Bragança Paulista, 21(1), 49-61. Disponible en: http://www.scielo.br/pdf/pusf/v21n1/2175-3563-pusf21-01-00049.pdf

11. Florenzano, R., Valdés, M., Cáceres E., Santander, S., Aspillaga C., \& Musalem, C. (2011). Relación entre ideación suicida y estilos parentales en un grupo de adolescentes chilenos. Revista Médica de Chile, 139, 1529-1533. Disponible en: http://www.academia.edu/1416579/Relaci\%C3\%B3n_entre_ideaci\% C3\%B3n_suicida_y_estilos_parentales_en_un_grupo_de_adolescente s_chilenos

12. Gaeta, L., \& Galvanovskis, A. (2009). Asertividad: Un análisis teórico-empírico. Enseñanza e Investigación en Psicología, 14(2), 
403-425.

Disponible

en:

http://file.redalyc.org/articulo.oa?id=29211992013

13. García, M., Cabanillas, G., Morán, V., \& Olaz, F. O. (2014). Diferencias de género en estudiantes universitarios de Argentina. Anuario Electrónico de Estudios en Comunicación Social, 7(2), 114134. http://revistas.urosario.edu.co/index.php/disertaciones/article/view/37 36.

14. García, M., García, A., \& Casanova, P. (2014). Prácticas educativas paternas que predicen la agresividad evaluada por distintos informantes. Revista Latinoamericana de Psicología, 46(3), 198-210. Disponible en: http://file.redalyc.org/articulo.oa?id=80533065007.

15. González, E., Molina, T., Montero, A., \& Martínez, V. (2013). Factores asociados al inicio sexual en adolescentes de ambos sexos de nivel socioeconómico medio-bajo de la Región Metropolitana. Revista Chilena de Obstetricia y Ginecología, 78(1), 4-13. Disponible en: http://revistasochog.cl/articulos/ver/598

16. Herrera, A., \& Repetto, P. (2014). Prácticas parentales y conductas de riesgo del peatón en una muestra de adolescentes chilenos. Revista Médica de Chile, 142, 975-981. Disponible en : http://www.scielo.cl/scielo.php?script=sci_arttext\&pid=S003498872014000800004\&lng=en\&nrm=iso\&tlng=en

17. Infante, A., \& Martínez, J. (2016). Concepciones sobre la crianza: El pensamiento de madres y padres de familia. Liberabit, 22(1), 31-41. Disponible en: http://www.scielo.org.pe/scielo.php?script=sci_arttext\&pid=S172948272016000100003\&lng=es\&nrm=iso

18. Lara, C. \& Silva, T. (2002). Estandarización de la Escala de Asertividad de Michelson y Wood en Niños y Adolescentes: II. Tesis inédita de licenciatura. Facultad de Psicología. México: Universidad Nacional Autónoma de México.

19. Malander, N. (2016). Percepción de prácticas parentales y estrategias de aprendizaje en estudiantes secundarios. Revista de Psicología, 25(1), 1-19. Disponible en: http://www.redalyc.org/articulo.oa?id=26446630004.

20. Michelson, L., \& Wood, R., (1982). Development and psychometric properties of the Children's Assertive Behaviour Scale. Journal of Behavioral Assessment, 4, 3-14. DOI:10.1007/BF01321378. Disponible en : https://link.springer.com/article/10.1007/BF01321378 
21. Neves, S., Xavier, A., Vieira, M., \& Rubin, K. (2013). Externalizing and internalizing problems: Contributions of attachment and parental practices. Psicologia: Reflexão e Crítica, 26(3), 617-625. Disponible en : http://dx.doi.org/10.1590/S0102-79722013000300022.

22. Nunes, S., Faraco, A., \& Vieira, M. (2013). Attachment and parental practices as predictors of behavioral disorders in boys and girls. Paidéia (Ribeirão Preto), 23(56), 369-377. DOI:10.1590/1982$43272356201311 . \quad$ Disponible en : https://www.researchgate.net/publication/281745686_Apego_e_Prati cas_Parentais_Como_Preditores_de_Disturbios_de_Comportamento_ em_Meninos_e_Meninas_Attachment_and_Parental_Practices_as_Pr edictors_of_Behavioral_Disorders_in_Boys_and_Girls

23. Oliva, A., Parra, A. \& Arranz, E. (2008). Estilos relacionales parentales y ajuste adolescente. Infancia y Aprendizaje, 31, 93-106. DOI: $10.1174 / 021037008783487093$

24. Pulido, S., Castro-Osorio, J., Peña, M., \& Ariza-Ramírez, D. (2013). Pautas, creencias y prácticas de crianza relacionadas con el castigo y su transmisión generacional. Revista Latinoamericana de Ciencias Sociales Niñez y Juventud, 11(11), 245-259. Disponible en: http://revistalatinoamericanaumanizales.cinde.org.co/wpcontent/uploads/2013/04/Pautas_creencias_a16vol111.pdf

25. Triana, A., Ávila, L., \& Malagón, A. (2010). Patrones de crianza y cuidado de niños y niñas en Boyacá. Revista Latinoamericana de Ciencias Sociales Niñez y Juventud, 8(2), 933-945. Disponible en: https://issuu.com/revistalcsnj/docs/revista_vol_11_n__1_act/260

26. Vega, C., González, F., Anguiano, S., Nava, C., \& Soria, R. (2009). Habilidades sociales y estrés infantil. Journal of Behavior, Health \& Social Issues, 1(1), 7-13. Disponible en: http://www.redalyc.org/pdf/2822/282221718001.pdf.

27. Vite, A., \& Pérez, M. (2014). Mediación de los esquemas cognitivos maternos y estilos parentales en la relación entre prácticas de crianza y problemas de comportamiento infantil. Avances en Psicología Latinoamericana, 32(3), 389-402. Disponible en: http://revistas.urosario.edu.co/index.php/apl/article/view/2693 